\title{
An Iterative Decomposed Piecewise-Linear Model Description
}

\author{
Victor Jimenez-Fernandez, Luis Hernandez-Martinez, and Arturo Sarmiento-Reyes
}

Electronics Department, National Institute for Astrophysics, Optics and Electronics, 72840 Tonantzintla, Puebla, Mexico

Correspondence should be addressed to Luis Hernandez-Martinez, luish@inaoep.mx

Received 2 March 2009; Accepted 3 June 2009

Recommended by Fahrettin Yakuphanoglu

A model description for the representation of one-dimensional piecewise-linear characteristics is presented. The model can be denoted as a decomposed one, because the independent and dependent variables of the PWL characteristic are treated separately. It is also called iterative, because the particular representation of each segment of the PWL characteristic depends on the value of only one parameter included in the mathematical formulation, it gives the possibility of modeling both, univalued and multivalued PWL characteristics.

Copyright (c) 2009 Victor Jimenez-Fernandez et al. This is an open access article distributed under the Creative Commons Attribution License, which permits unrestricted use, distribution, and reproduction in any medium, provided the original work is properly cited.

\section{Introduction}

In order to be able to simulate nonlinear networks, the nonlinear behavior of the components must be modeled first. The nonlinear behavior cannot exactly be described by familiar analytical nonlinear functions, so it must be approximated by a known function. Some approaches to overcome this problems are the polynomial approximation, table-look-up technique, and PWL approximation.

A lot of research has been done in the area of approximating nonlinear functions using polynomials as well as in the field of calculating transcendental functions. Applying such methods leads to simulators like SPICE [1].

The technique alá table-look-up consisting in storing several sample points of a nonlinear function in a table. If a function value is needed, the entry in the table is used or interpolated.

In PWL approximation, the nonlinear function is repeatedly approximated locally by a linear mapping. This results in a large collection of linear mappings which can be stored in a compact closed form model $[2,3]$. These models have been applied in the so-called PWL simulators [4-6].

There are many important proposals in the area of PWL modeling, but no best model description can be found because the advantages or disadvantages of any PWL model depends on its application [7].

The main contributions of the PWL model, which in Section 2 will be exposed, are the possibility of describing all kinds of one-dimensional PWL characteristics (univalued and multivalued) having mathematical formulation which is not so heavy to be computed because it is based on an iterative system of linear equations, the advantage of obtaining the model parameters directly from the graphic coordinates and the simulation capability of the model, because it is compatible with iterative simulation techniques [8].

The model is denoted as decomposed, because the independent variable $x$ and the dependent variable $y=$ $f(x)$ are included separately in a system of linear equations. The model is also denoted as iterative, because there is a parameter $k$, which selects a specific segment from the set of $L$-segments that constitutes the complete PWL characteristic.

\section{Model Description}

The PWL characteristic, depicted in Figure 1, represents a general one-dimensional function $y=f(x)$ characterized by $L$ linear segments and $L+1$ coordinates: $\left(X_{1}, Y_{1}\right),\left(X_{2}, Y_{2}\right), \ldots,\left(X_{L+1}, Y_{L+1}\right)$.

An iterative decomposed formulation for this characteristic can be obtained as follows.

Firstly, the particular problem of obtaining the line equation for any of the $k$ th segments included in the PWL characteristic is analyzed. The graphic exposition of this problem is depicted in Figure 2. 


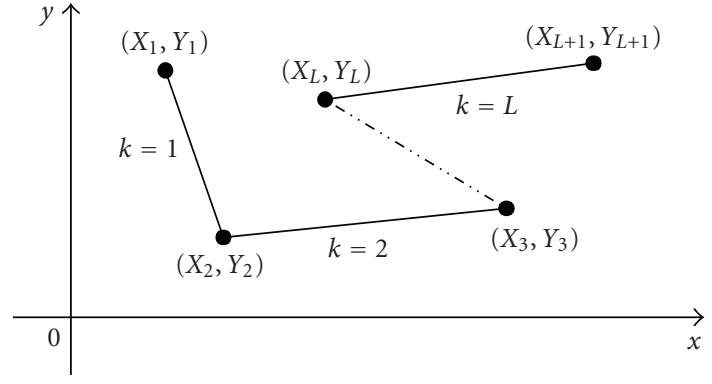

FIGURE 1: PWL characteristic and its graphic coordinates.

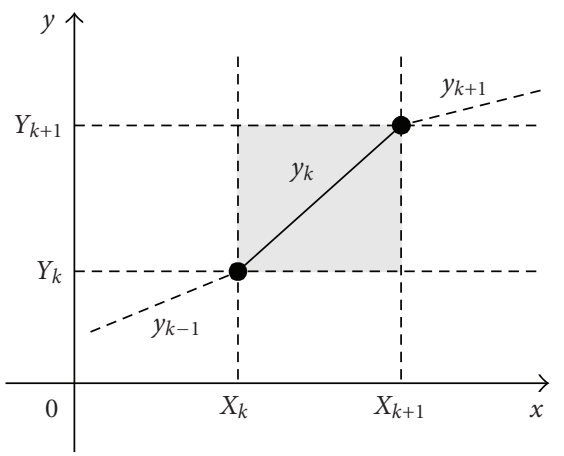

FIgURE 2: A $k$ th segment belonging to a PWL characteristic.

The line equation for the $k$ th segment is given as

$$
y_{k}=\left(\frac{Y_{k+1}-Y_{k}}{X_{k+1}-X_{k}}\right) x+\left(\frac{Y_{k} X_{k+1}-Y_{k+1} X_{k}}{X_{k+1}-X_{k}}\right) .
$$

Then, (1) is factored into the form

$$
y_{k}=P_{k} Y_{k}+Q_{k} Y_{k+1},
$$

where $P_{k}$ and $Q_{k}$ are variables defined by the determinant relations:

$$
P_{k}=\frac{\left|\begin{array}{cc}
x & X_{k+1} \\
1 & 1
\end{array}\right|}{\left|\begin{array}{cc}
X_{k} & X_{k+1} \\
1 & 1
\end{array}\right|}, \quad Q_{k}=\frac{\left|\begin{array}{cc}
X_{k} & x \\
1 & 1
\end{array}\right|}{\left|\begin{array}{cc}
X_{k} & X_{k+1} \\
1 & 1
\end{array}\right|} .
$$

The relation between (2) and (3) is expressed as the system of linear equations given in

$$
\left[\begin{array}{c}
y_{k} \\
x \\
1
\end{array}\right]=\left[\begin{array}{cc}
Y_{k} & Y_{k+1} \\
X_{k} & X_{k+1} \\
1 & 1
\end{array}\right]\left[\begin{array}{l}
P_{k} \\
Q_{k}
\end{array}\right] .
$$

The above linear system represents the $k$ th line equation included in the PWL characteristic to be modeled. Finally, a complete mathematical expression which assures a description for the PWL characteristic depicted in Figure 1 is obtained if (4) is generalized for all $L$ segments. Thus, the parametric model description can be written:

$$
\left[\begin{array}{l}
y \\
x \\
1
\end{array}\right]=\sum_{i=1}^{L}\left[\begin{array}{cc}
Y_{i} & Y_{i+1} \\
X_{i} & X_{i+1} \\
1 & 1
\end{array}\right]\left[\begin{array}{c}
P_{i} \\
Q_{i}
\end{array}\right] C_{i}(k)
$$

with the condition

$$
Q_{i}(x), P_{i}(x), Q_{i}(y), P_{i}(y) \geq 0,
$$

where $C_{i}(k)$ is denoted as activation vector and it is defined as follows:

$$
C_{i}(k)=\frac{1}{i !(L-i) !} \prod_{m=0}^{i-1}|k-m| \prod_{n=1}^{L-i}|k-(i+n)|
$$

for $i=1, \ldots, L-1$, and

$$
C_{i}(k)=\frac{1}{L !} \prod_{r=0}^{L-1}|k-r|
$$

for $i=L$.

The variables $Q_{i}$ and $P_{i}$ in (5) have an important function because they control the interval where a $k$ th line equation exists. This is expressed in the condition (6) and it is demonstrated as follows.

Firsty, the notations $\Delta X_{i}$ and $\Delta Y_{i}$ are defined as

$$
\Delta X_{i}=X_{i+1}-X_{i}, \quad \Delta Y_{i}=Y_{i+1}-Y_{i}
$$

Then, mathematical expressions, for the variables $P_{i}$ and $Q_{i}$, can be obtained if the following decomposed linear equations, which are included in (5), are algebraically manipulated:

$$
\begin{gathered}
y=Y_{i} P_{i}+Y_{i+1} Q_{i}, \\
x=X_{i} P_{i}+X_{i+1} Q_{i}, \\
1=P_{i}+Q_{i} .
\end{gathered}
$$

Isolating $P_{i}$ from (12) and substituting it into (10), (11), $Q_{i}$ can be expressed as

$$
Q_{i}(x)=\frac{x}{\Delta X_{i}}-\frac{X_{i}}{\Delta X_{i}}, \quad Q_{i}(y)=\frac{y}{\Delta Y_{i}}-\frac{Y_{i}}{\Delta Y_{i}} .
$$

A similar analysis where $Q_{i}$ is isolated from (12) and then susbstituted into (10), (11) yields

$$
P_{i}(x)=\frac{-x}{\Delta X_{i}}+\frac{X_{i+1}}{\Delta X_{i}}, \quad P_{i}(y)=\frac{-y}{\Delta Y_{i}}+\frac{Y_{i+1}}{\Delta Y_{i}} .
$$

If (13) and (14) are subjected to the exposed condition in (6) results

$$
\begin{aligned}
& \Delta X_{i} Q_{i}(x)=x-X_{i} \geq 0, \quad x \geq X_{i}, \\
& \Delta Y_{i} Q_{i}(y)=y-Y_{i} \geq 0, \quad y \geq Y_{i}, \\
& \Delta X_{i} P_{i}(x)=X_{i+1}-x \geq 0, \quad x \leq X_{i+1}, \\
& \Delta X_{i} P_{i}(y)=Y_{i+1}-y \geq 0, \quad y \leq Y_{i+1} .
\end{aligned}
$$

The inequations (15) indicate the intervals where a linear segment belonging to a PWL characteristic exists. The shadow area depicted in Figure 2 results by the intersection of these intervals for the $k$ th PWL segment. 


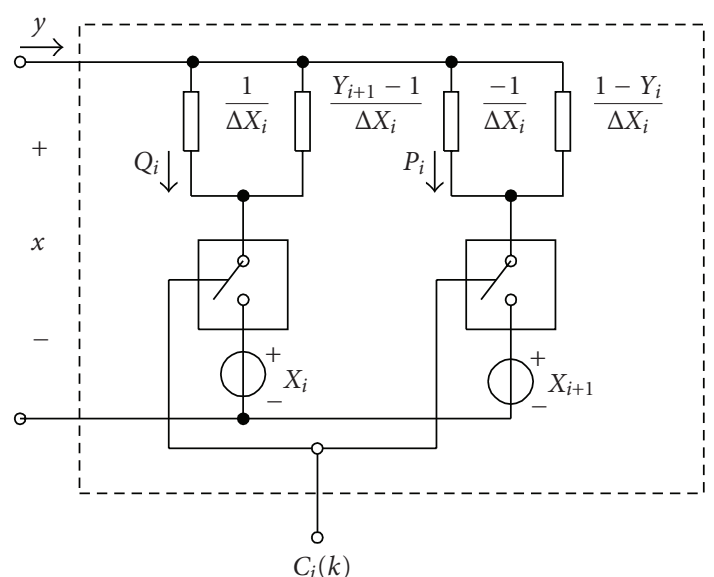

Figure 3: Equivalent circuit for a PWL segment.

\begin{tabular}{|c|c|}
\hline Element & Symbol \\
\hline $\begin{array}{c}\text { Switch } \\
\text { controlled by } \\
C_{n}(k)\end{array}$ & \\
\hline
\end{tabular}

FIGURE 4: Switch symbol included in the equivalent circuit diagram.

TABle 1: $\left(V_{i}, I_{i}\right)$ coordinates for the PWL characteristic of Figure 5(b).

\begin{tabular}{lccc}
\hline$i$ & Coordinates & $V_{i}$ & $I_{i}$ \\
\hline 1 & B1 & 0 & 0 \\
2 & B2 & 3 & 3 \\
3 & B3 & 1 & 4 \\
4 & B 4 & 4 & 1 \\
\hline
\end{tabular}

\section{Electrical Model}

The linear system in (5) can be electrically modeled by the circuit shown in Figure 3. In this circuit, the variables $Q_{i}$ and $P_{i}$ have a physical meaning, and represent the current flow through the conductances whose values are $\left(\Delta X_{i}\right)^{-1}$ and $-\left(\Delta X_{i}\right)^{-1}$, respectively. The electrical diagram includes two switches which are controlled simultaneously by $C_{i}(k)$. Figure 4 shows the symbol used to represent them. On the one hand, it can be seen that when the switches are open, these variables are equal to zero, it occurs when $C_{i}(k)=0$, on the other hand, when $C_{i}(k)=1$ the switches are closed and the variables (currents $Q_{i}$ and $P_{i}$ ) are different to zero.

\section{Example}

Consider the theoretical nonlinear element and its PWL characteristic depicted in Figure 5. Determine their iterative decomposed model description.

An analysis from Figure 5(b) shows the existence of three linear equations labeled as (1), (2), and (3). The four

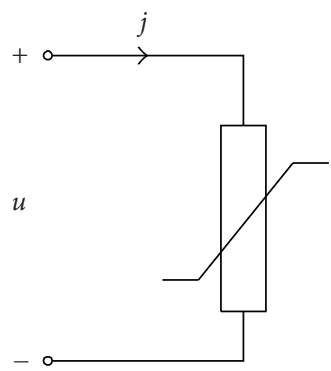

(a)

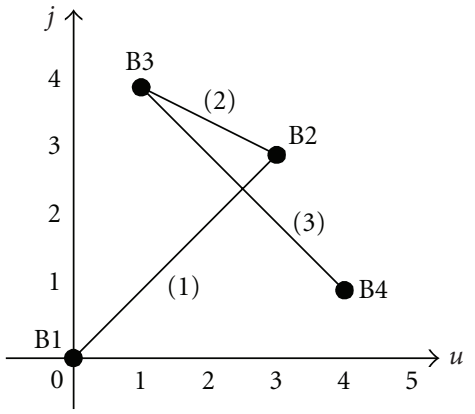

(b)
FIgURE 5: (a) Nonlinear element, (b) PWL characteristic for the nonlinear element.

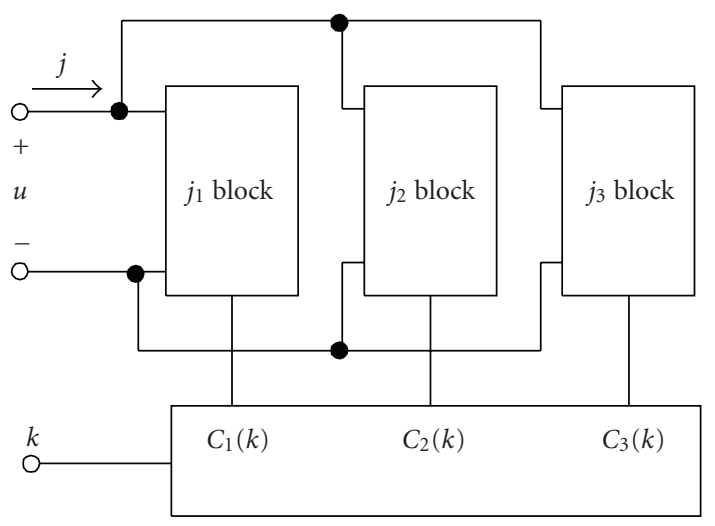

Figure 6: Electrical circuit model for the Example.

graphic coordinates are designated as $\mathrm{B} 1, \mathrm{~B} 2, \mathrm{~B} 3$, and $\mathrm{B} 4$, and summarized in Table 1.

There are three segments, therefore $L=3$ and $k=1,2,3$. Substituting $L$ and the $\left(V_{i}, I_{i}\right)$ coordinates of Table 1 into (5), results:

$$
\begin{aligned}
{\left[\begin{array}{l}
j \\
u \\
1
\end{array}\right]=} & \sum_{i=1}^{3}\left[\begin{array}{cc}
I_{i} & I_{i+1} \\
V_{i} & V_{i+1} \\
1 & 1
\end{array}\right]\left[\begin{array}{c}
P_{i} \\
Q_{i}
\end{array}\right] C_{i}(k) \\
= & {\left[\begin{array}{ll}
0 & 3 \\
0 & 3 \\
1 & 1
\end{array}\right]\left[\begin{array}{l}
P_{1} \\
Q_{1}
\end{array}\right] C_{1}(k)+\left[\begin{array}{ll}
3 & 4 \\
3 & 1 \\
1 & 1
\end{array}\right]\left[\begin{array}{l}
P_{2} \\
Q_{2}
\end{array}\right] C_{2}(k) } \\
& +\left[\begin{array}{ll}
4 & 1 \\
1 & 4 \\
1 & 1
\end{array}\right]\left[\begin{array}{l}
P_{3} \\
Q_{3}
\end{array}\right] C_{3}(k) .
\end{aligned}
$$

Substituting $L$ in (7) and (8), the terms of the activation vector $C_{i}(k)$ are given by

$$
\begin{aligned}
& C_{1}(k)=\frac{k|k-2||k-3|}{2}, \\
& C_{2}(k)=\frac{k|k-1||k-3|}{2}, \\
& C_{3}(k)=\frac{k|k-1||k-2|}{6} .
\end{aligned}
$$




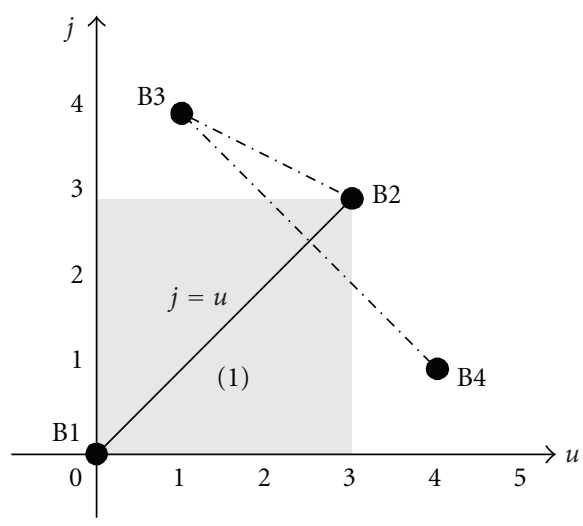

FIgURE 7: Interval intersection for $k=1$.

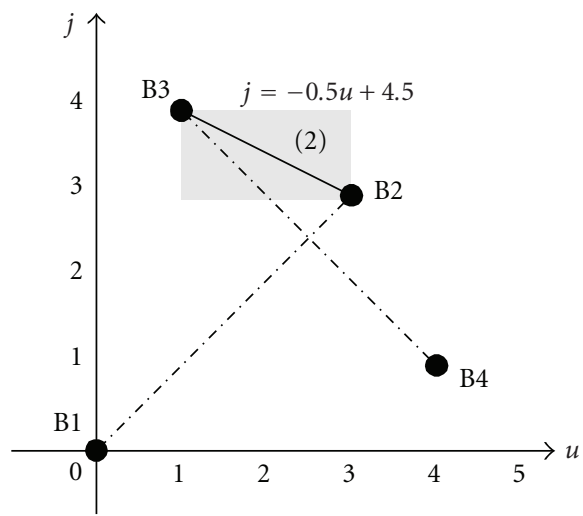

FIGURE 8: Interval intersection for $k=2$.

Figure 6 shows the electrical circuit which models the PWL characteristic analyzed in this example. Each $j_{k}$ th Block is a simplified representation of the circuit shown in Figure 3.

When $k=1, C_{1}(k)=1, C_{2}(k)=0, C_{3}(k)=0$, and the resulting linear system is

$$
\left[\begin{array}{l}
j \\
u \\
1
\end{array}\right]=\left[\begin{array}{ll}
0 & 3 \\
0 & 3 \\
1 & 1
\end{array}\right]\left[\begin{array}{l}
P_{1} \\
Q_{1}
\end{array}\right]+0+0
$$

The line equation is $j=u$ bounded by the intervals $u=[0,3]$ and $j=[0,3]$.

When $k=2, C_{1}(k)=0, C_{2}(k)=1, C_{3}(k)=0$, and the resulting linear system is

$$
\left[\begin{array}{l}
j \\
u \\
1
\end{array}\right]=0+\left[\begin{array}{ll}
3 & 4 \\
3 & 1 \\
1 & 1
\end{array}\right]\left[\begin{array}{l}
P_{2} \\
Q_{2}
\end{array}\right]+0
$$

The line equation is $j=-0.5 u+4.5$ bounded by the intervals $u=[1,3]$ and $j=[3,4]$.

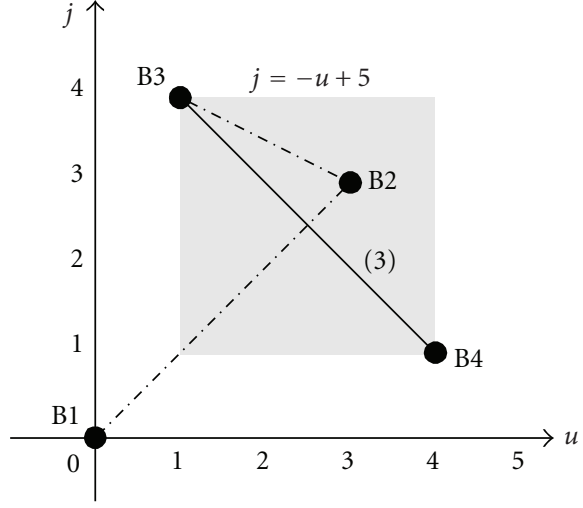

FIGURE 9: Interval intersection for $k=3$.

TABLE 2: $Q_{k}$ and $P_{k}$ variables subjected by the conditions (6).

\begin{tabular}{cccc}
\hline$k$ & $Q_{k}$ & $P_{k}$ & Interval \\
\hline 1 & $u / 3 \geq 0$ & $1-u / 3 \geq 0$ & $0 \leq u \leq 3$ \\
& $j / 3 \geq 0$ & $1-j / 3 \geq 0$ & $0 \leq j \leq 3$ \\
\hline \multirow{2}{*}{2} & $-u / 2+3 / 2 \geq 0$ & $u / 2-1 / 2 \geq 0$ & $1 \leq u \leq 3$ \\
& $j-3 \geq 0$ & $-j+4 \geq 0$ & $3 \leq j \leq 4$ \\
\hline \multirow{2}{*}{3} & $u / 3-1 / 3 \geq 0$ & $-u / 3+4 / 3 \geq 0$ & $1 \leq u \leq 4$ \\
& $-j / 3+4 / 3 \geq 0$ & $j / 3-1 / 3 \geq 0$ & $1 \leq j \leq 4$ \\
\hline
\end{tabular}

When $k=3, C_{1}(k)=0, C_{2}(k)=0, C_{3}(k)=1$, and the resulting linear system is

$$
\left[\begin{array}{l}
j \\
u \\
1
\end{array}\right]=0+0+\left[\begin{array}{ll}
4 & 1 \\
1 & 4 \\
1 & 1
\end{array}\right]\left[\begin{array}{l}
P_{3} \\
Q_{3}
\end{array}\right] .
$$

The line equation is $j=-u+5$ bounded by the intervals $u=[1,4]$ and $j=[1,4]$.

The above results are summarized in Table 2 and Figures 7,8 , and 9 .

\section{Conclusions}

The proposed iterative and decomposed model can describe any arbitrary PWL function (univalued or multivalued) by considering as model parameters its graphic coordinates. The interval in the model bounds the range where a linear segment is valid and it is determined by the variables $Q_{i}$ and $P_{i}$. These variables have a physical meaning (currents) in the electrical model. The equivalent circuit, of the proposed PWL description, has simulation capability because it is compatible with iterative simulation techniques.

\section{Acknowledgments}

Victor Jimenez-Fernandez is holder of a scholarship from CONACyT-México by the Contract 183249. This work has been partially supported by a CONACyT-México research project under Grant 60261. 


\section{References}

[1] A. Vladimirescu and S. Liu, "The simulation of MOS integrated circuits SPICE2," Memorandum no. UCB/ERL M80/7, Electronics Research Laboratory, College of Engineering, University of Berkeley, Berkeley, Calif, USA, February 1980.

[2] L. O. Chua and S. M. Kang, "Section-wise piecewise-linear functions: canonical representation, properties, and applications," Proceedings of the IEEE, vol. 65, no. 6, pp. 915-929, 1977.

[3] W. M. G. van Bokhoven, Piecewise Linear Modeling and Analysis, Kluwer Technische Boeken, Deventer, The Netherlands, 1981.

[4] J. T. J. van Eindhoven, A piecewise linear simulator for large scale integrated circuits, Ph.D. dissertation, Eindhoven, The Netherlands, 1984.

[5] T. A. M. Kevenaar and D. M. W. Leenaerts, "A flexible hierarchical piecewise linear simulator," Integration, the VLSI Journal, vol. 12, no. 2, pp. 211-235, 1991.

[6] M. T. van Stiphout, PLATO: a piecewise-linear analysis tool for mixed level circuit simulation, Ph.D. dissertation, Technische Universiteit Eindhoven, Eindhoven, The Netherlands, 1990.

[7] T. A. M. Kevenaar and D. M. W. Leenaerts, "A comparison of piecewise-linear model descriptions," IEEE Transactions, CAS1: Fundamental Theory and Applications, vol. 39, no. 12, pp. 996-1004, 1992.

[8] L. O. Chua, "Analysis and synthesis of multi-valued memoryless nonlinear networks," IEEE Transactions on Circuit Theory, vol. 14, no. 2, pp. 192-209, 1967. 

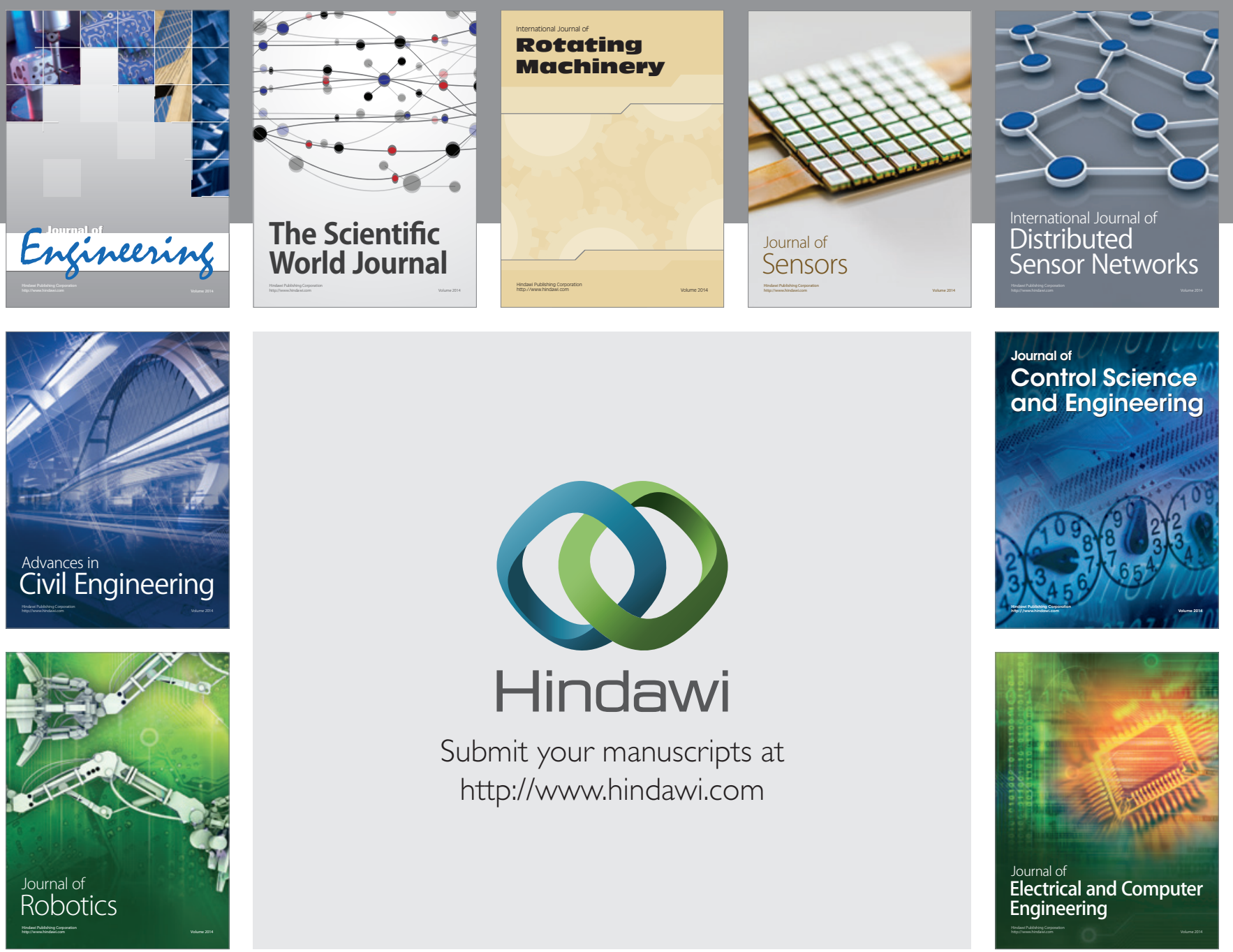

Submit your manuscripts at

http://www.hindawi.com
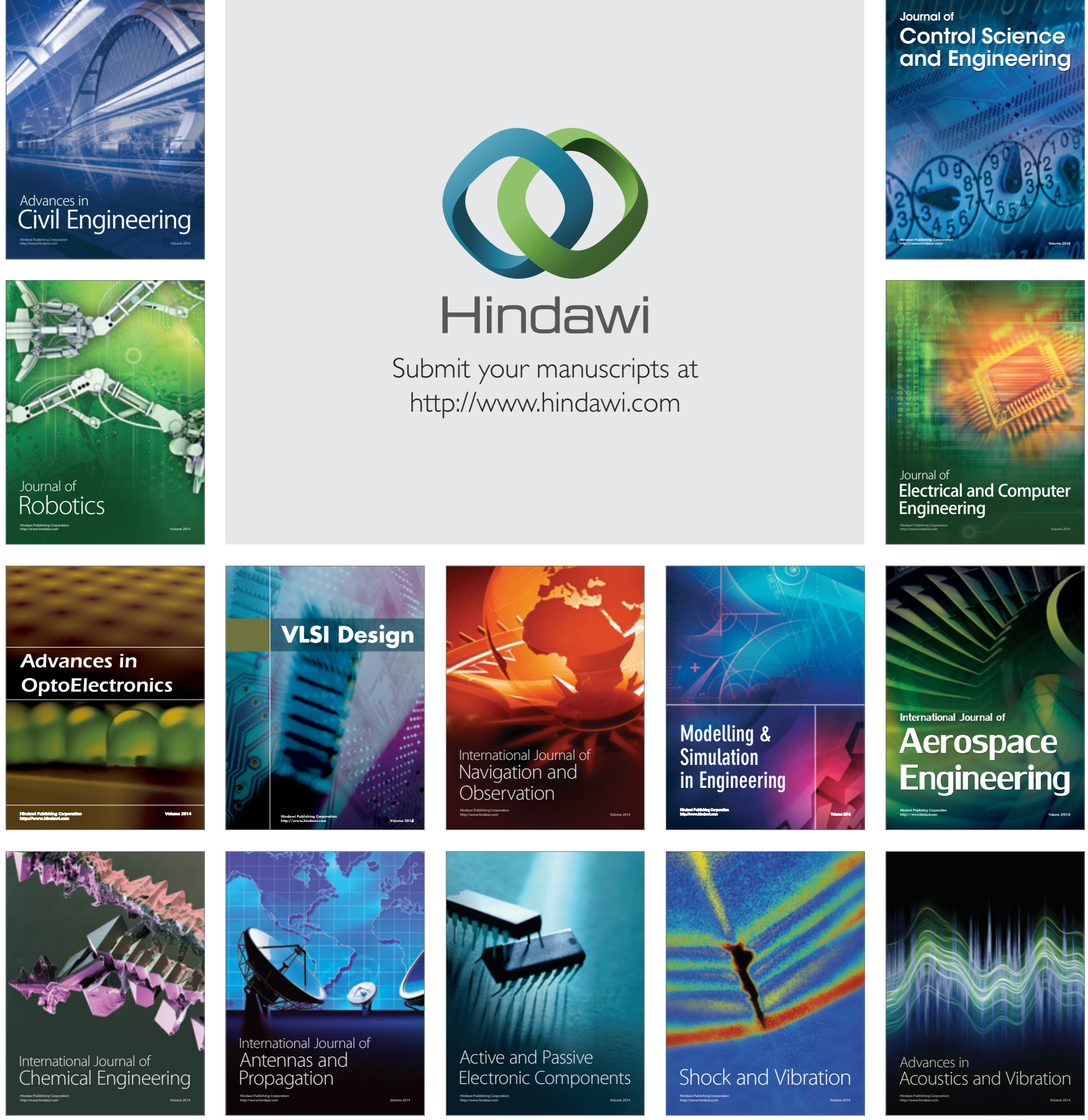